\title{
Striking regression of temporal lobe necrosis following radiotherapy for nasopharyngeal carcinoma in a clinical trial of anti-mycobacterium therapy
}

\author{
Yanqing Feng ${ }^{1}$, Weixi Zhang ${ }^{1}$, Xi Chen ${ }^{2}$, Zhenhua Gao ${ }^{3}$, Minying Zheng ${ }^{1}$, Ning Guo ${ }^{1}$, \\ Fan Huang $^{1}$, Ke Ma ${ }^{1}$ \\ ${ }^{1}$ Department of Neurology, The First Affiliated Hospital of Sun Yat-sen University, Guangzhou, PR China \\ ${ }^{2}$ Department of Rehabilitation Medicine, The First Affiliated Hospital of Sun Yat-sen University, Guangzhou, PR China \\ ${ }^{3}$ Department of Radiology, The First Affiliated Hospital of Sun Yat-sen University, Guangzhou, PR China
}

\author{
Email address: \\ fyqgz@sina.com(Yanqing Feng)
}

\section{To cite this article:}

Yanqing Feng, Weixi Zhang, Xi Chen, Zhenhua Gao, Minying Zheng, Ning Guo, Fan Huang, Ke Ma. Striking Regression of Temporal Lobe Necrosis Following Radiotherapy for Nasopharyngeal Carcinoma in a Clinical trial of Anti-Mycobacterium Therapy. Journal of Cancer Treatment and Research. Vol. 2, No. 5, 2014, pp. 48-55. doi: 10.11648/j.jctr.20140205.12

\begin{abstract}
The mechanism of radiation-induced delayed brain injuries remains poorly understood, and few treatment options are available. The aim of this study was to investigate whether temporal lobe necrosis can be reversed by anti-mycobacterium therapy (AMT). We conducted this prospective, controlled study in southern China. Ten patients with symptomatic delayed radiation injury were monitored during AMT and compared with a control group of 11 patients who received current standard therapies. Activities of daily living were assessed by the Barthel Index (BI) at study entry and after 2 years of therapy. Magnetic resonance imaging (MRI) was performed before treatment, and changes were monitored during the study. Kaplan-Meier analysis was employed to delineate time-related mortality. A significant treatment effect was observed in the AMT group. The patients' headaches, seizures, dizziness, and cognitive deterioration rapidly improved. BI improved in the AMT treatment group compared with the control group after 2 years (Mann-Whitney $U$ test; $P=0 \cdot 001$ ). Abnormalities of the temporal lobes, observed by MRI, markedly decreased over time in eight patients, whereas in the control group significant BI deterioration was observed (Wilcoxon signed-rank test; $P=0.003$ ) and the patients did not show favorable MRI changes. By 24 months, there was a significant difference between the AMT and control groups with respect to survival time (log-rank test; $P=0 \cdot 011$ ). The results of the present study suggest that radiation necrosis of the brain can be successfully managed by AMT. These findings must be confirmed in large, double-blind, randomized clinical trials.
\end{abstract}

Keywords: Radiation-Induced Delayed Brain Injuries, Anti-Mycobacterium Therapy, Temporal Lobe Necrosis, Reverse

\section{Introduction}

Radiotherapy is used to treat a wide variety of head and neck tumors that arise in and around the skull base. Radiation-induced injury may occur when adjacent normal nervous system tissue is exposed to radiation. Delayed radiation injury (DRI) of the normal brain can be a devastating complication and generally occurs months to years after the initiation of therapy. DRI is generally progressive and irreversible, and it has a tremendous negative impact on a patient's quality of life. ${ }^{1}$ Fischer and Holfelder initially described the delayed radiation-induced histological changes in $1930 .^{2}$ Although many scientific studies of DRI have been conducted, the pathophysiological mechanism of DRI remains poorly understood. ${ }^{3}$ To date, the treatment for DRI has typically involved management of symptoms. Although spontaneous resolution of small white matter lesions (WMLs) has been reported occasionally, ${ }^{4}$ clinicians have not found an effective method to stop the progression of necrosis, which appears as cysts and/or contrast-enhanced lesions on brain magnetic resonance imaging (MRI) developed after WMLs. Data from surgical resection and biopsy have shown that histologic features of radiation injury are diverse and not specific to radiation. Classically, however, the most prominent histopathological changes are reactive white matter edema, demyelination, coagulative necrosis, and cysts with central liquefaction and gliosis in the walls. ${ }^{5,6}$ The presence of these histological changes may be indicative of DRI, a result of 
some type of inflammation induced by radiation.

Temporal lobe necrosis (TLN) after external radiation is one of the most dreaded DRI complications in nasopharyngeal carcinoma. $^{7,8}$ Evaluation of the temporal lobes in patients previously treated for nasopharyngeal carcinoma can provide a better understanding of DRI in the brain unaffected by the underlying tumor and outside of the planned field of radiation. In a study by Cheng et al., ${ }^{9} 20 \%$ of the patients undergoing surgery for radiation necrosis were found to have a cerebral abscess, indicating that TLN might be due to radiation-induced inflammation. That study demonstrated that the response to antibiotic therapy is not satisfactory in DRI, even with a pathologically proven abscess. By chance, we discovered that anti-mycobacterium therapy (AMT) affects TLN. In 2008, we used AMT to treat a symptomatic TLN patient with symptoms of headache and dysphasia, accompanied by pulmonary tuberculosis as evidenced by a positive chest X-ray test. AMT provided unexpected symptom relief from TLN. Based on this experience, we developed a trial protocol to evaluate whether AMT can reverse TLN with respect to both symptoms and MRI abnormalities. This information can be used for future understanding, awareness, prevention, and management by both physicians and patients.

\section{Patients and Methods}

\subsection{Study Design}

This prospective, open-labeled and controlled nonrandomized study was conducted between January 2009 and October 2013. Twenty-one patients qualified for inclusion in the study, according to the inclusion and exclusion criteria (see below). There were 16 males and 5 females. Patients who fulfilled the following eligibility criteria were recruited in this study: (i) histopathologically proven nasopharyngeal carcinoma with radiation therapy and negative brain MRI before radiation therapy; (ii) cranial irradiation $\geq 6$ months prior to study entry; (iii) MRI evidence of bilateral WMLs and/or cysts with or without foci of contrast enhancement on MRI; and (iv) evidence of progressive neurologic signs or symptoms related to TLN. The following exclusion criteria were used: (i) significant hepatic or renal insufficiency; (ii) evidence of brain metastasis, brain abscess, any intracranial tumor, cerebral infarction, or demyelinating disease; or (iii) clinical manifestations, such as fever or meningeal irritation signs indicating meningitis.

The patients were categorized into the different groups based on the following considerations: (i) consent to receive AMT treatment and (ii) consent to stop steroid therapy. Ten patients were included in the treatment group, and 11 patients (control group) underwent standard therapies.

\subsection{Planned Interventions (Treatment) and Follow-Up}

Eligible patients underwent complete physical and neurological examinations upon entry. Upon admission, pretreatment contrast-enhanced MRI of the brain was performed on all of the patients. In the active treatment group, all treatments with corticosteroids were discontinued for patients prior to AMT initiation. Our initial treatment protocols (Treatment Phase 1) comprised amikacin (600 $\mathrm{mg}$ /day) and mannitol $125 \mathrm{ml}$ (bid) for 2 weeks, together with a three-drug AMT regimen (per day: isoniazid $8 \mathrm{mg} / \mathrm{kg}$, rifampicin $10 \mathrm{mg} / \mathrm{kg}$, and pyrazinamide $25 \mathrm{mg} / \mathrm{kg}$ ), followed by treatment with a three-drug AMT regimen for 9 months (Treatment Phase 2), and then treatment with a combination of isoniazid and rifampicin with the same dosages that ceased after 24 months (Treatment Phase 3). Treatment was carried out under extensive observation. When a worsened condition occurred during the Phase 2 period, short-term mannitol, amikacin, and/or low-dose intravenous immunoglobulin were used to improve the symptoms. All of the patients had weekly liver function tests for the first month of therapy and every 3 months thereafter.

In the control group, during the observational period treatments were conducted using routine regimens, including steroids, intravenous immunoglobulin, hyperbaric oxygen, nerve growth factor, and mannitol alone or in combination. Steroid treatment was defined as $10 \mathrm{mg}$ of dexamethasone (or equivalent) daily and was administered to patients with severe clinical symptoms and radiologic evidence of a mass effect. Each patient was followed and assessed by the same physician during the study.

\subsection{Outcome Measures}

Eligible patients underwent complete physical and neurological examinations upon entry, at 12 and 24 months. The clinical and radiological evaluations were not blinded. Activities of daily living were evaluated to assess the neurological status of the subjects. These were assessed using the Barthel Index (BI) (0-100 scale, with lower scores denoting less independence in activities of daily living) along with evaluation by MRI.

The university ethics committee approved the present study. All of the patients were informed of the potential short- and long-term drug complications of AMT. Written informed consent was obtained from patients who agreed to participate in the study. The patients were instructed to contact the study neurologist in the event of neurological symptoms.

The present study is registered with controlled-trials.com (number ISRCTN04023349).

\subsection{Statistical Analysis}

For the comparison of descriptive data, the Mann-Whitney $\mathrm{U}$ test was used for the analysis of variables that did not fit a normal distribution. The Wilcoxon signed-rank test was used for comparisons within groups. Fisher's exact test was used for categorical variables. Mortality was estimated by the Kaplan-Meier method, and results were compared between groups using the log-rank test. The Statistical Package for the Social Sciences (version 16·0; SPSS, Inc., Chicago, IL, USA) was used to perform these tests. A P value less than $0 \cdot 05$ was considered to indicate a statistically significant difference. 


\section{Results}

\subsection{Demographic and Clinical Characteristics}

The baseline clinical characteristics of all of the patients are listed in Table 1. The AMT and control groups were comparable for all of the baseline variables. Ten patients with TLN were included in the AMT group (age range, 39-60 years), and 11 patients were in the control group (38-63 years). All patients had undergone radiotherapy for nasopharyngeal carcinoma and showed no evidence of recurrent disease upon entering the trial. The main presentations were epilepsy, dizziness, headache, amnesia, cognitive dysfunction, and dysphasia.

\subsection{Control Group}

During the study period, all of the control patients showed worsening neurological signs and symptoms. The patients were treated with corticosteroids alone or in combination for symptom relief. Two patients were treated surgically at the Department of Neurosurgery due to symptom development, with poor outcomes. There were nine deaths in this group. One patient had marked deterioration and was bedridden. Another patient experienced chronically increasing difficulty in speaking and swallowing, without temporal lesion expansion apparent in an MRI.

\subsection{AMT Group}

Treatment was well tolerated. All patients receiving AMT showed improvement in neurological signs and symptoms in Treatment Phase 1, and the response occurred early in the course of treatment for all patients. Clinical worsening halted during the beginning of the 2-week course of active treatment. The rapidly improved symptoms included headaches, seizures, dizziness, and cognitive deterioration. Two TLN patients had difficulty swallowing, but their dysphasia gradually improved with the active treatment. Improvement during the treatment was sustained throughout follow-up in seven patients.

The other three who had initial treatment successes experienced worsening in Treatment Phase 2. One patient suffered worsening symptoms after 8 months of AMT and was then successfully treated with combination therapy of mannitol and intravenous immunoglobulin in the hospital. Nevertheless, another two patients suffered treatment failure and died. One of two patients suffered anorexia and weakness after 2 months of treatment, with expanding temporal lesions on MRI. This patient refused to take any medicine or be hospitalized and died 2 weeks later. Another patient experienced dizziness and difficulty in walking after 3 months of treatment, was diagnosed with temporal lobe herniation at another hospital, and died 1 month after.

The treatment also led to improvement in non-neurological complications, including xerostomia, foul odor, and trismus in the AMT group.

Evaluation of activities of daily living showed that BI improved in the AMT treatment group compared with the control group after 2 years (Mann-Whitney $\mathrm{U}$ test; $\mathrm{P}=0 \cdot 001$ ).
However, in the control group, significant BI deterioration was observed (Wilcoxon signed-rank test; $\mathrm{P}=0 \cdot 003$; Table 1).

Table 1. Demographic and baseline characteristics.

\begin{tabular}{llll}
\hline Characteristic & AMT group & Control group & P value \\
\hline $\begin{array}{l}\text { Sex: M/F } \\
\begin{array}{l}\text { Mean age at study } \\
\text { entry, years (range) }\end{array}\end{array}$ & $3 / 7$ & $2 / 9$ & $0 \cdot 635 \mathrm{a}$ \\
$\begin{array}{l}\text { Median disease } \\
\text { duration, years (IQR) }\end{array}$ & $3 \cdot 6(2 \cdot 0-4 \cdot 25)$ & $3 \cdot 3(1-4 \cdot 0)$ & $0 \cdot 322 \mathrm{~b}$ \\
$\begin{array}{l}\text { Total dosage of } \\
\text { radiation treatment, Gy }\end{array}$ & $70 \cdot 9 \pm 7 \cdot 39$ & $71 \cdot 8 \pm 5 \cdot 67$ & $0 \cdot 431 \mathrm{~b}$ \\
$\begin{array}{l}\text { Barthel index at } \\
\text { enrollment }\end{array}$ & $75 \cdot 0 \pm 12 \cdot 3$ & $76 \cdot 8 \pm 9 \cdot 6$ & $0 \cdot 831 \mathrm{~b}$ \\
$\begin{array}{l}\text { Barthel index at year 1 } \\
\text { Barthel index at year 2 }\end{array}$ & $75 \cdot 0 \pm 40 \cdot 3$ & $28 \cdot 2 \pm 34 \cdot 9$ & $0 \cdot 007 \mathrm{~b}$ \\
\hline
\end{tabular}

M, male; F, female; AMT, anti-mycobacterium treatment

${ }^{\text {a }}$ Fisher's exact test (two-sided)

${ }^{\mathrm{b}}$ Mann-Whitney $U$ test

${ }^{c}$ Wilcoxon signed-rank test $(P=0 \cdot 003$, compared with enrollment $)$

\subsection{Kaplan-Meier Survival Analysis}

Two patients in the AMT group and nine patients in the control group died during the study period. Figure 1 shows the Kaplan-Meier estimates for the occurrence of death in AMT and control patients. The log-rank tests of the Kaplan-Meier analyses, considering the data at baseline, indicated significant differences between groups with and without AMT treatment $(\mathrm{P}=0 \cdot 011)$.

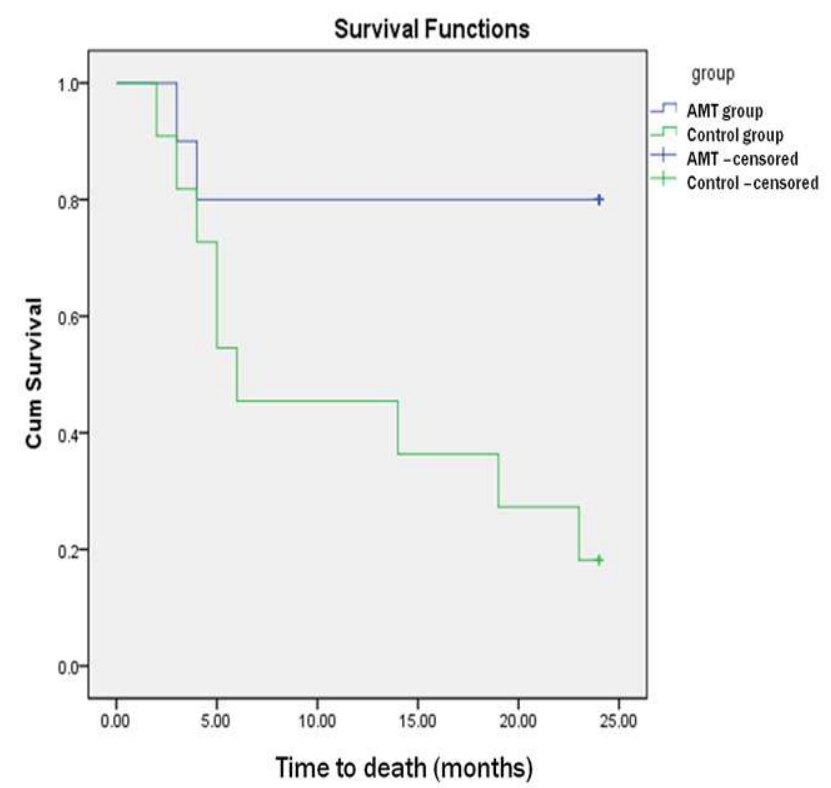

Fig. 1. Temporal lobe necrosis survival curve. Kaplan-Meier analysis showing the cumulative proportion of patients surviving after the follow-up period in the anti-mycobacterium treatment (AMT) and control groups. The result of the log-rank analysis was significant. AMT was associated with a lower mortality than the standard treatments.

\subsection{Efficacy as Measured by MRI}

During the study period, signal abnormalities in follow-up MRIs were markedly decreased in size and prominence by the end of AMT in eight patients. Characteristic imaging 
abnormalities of TLN, such as the typical "Swiss cheese" showed increasing size reductions (Fig. 2). pattern and necrotic masses with finger-like projections,

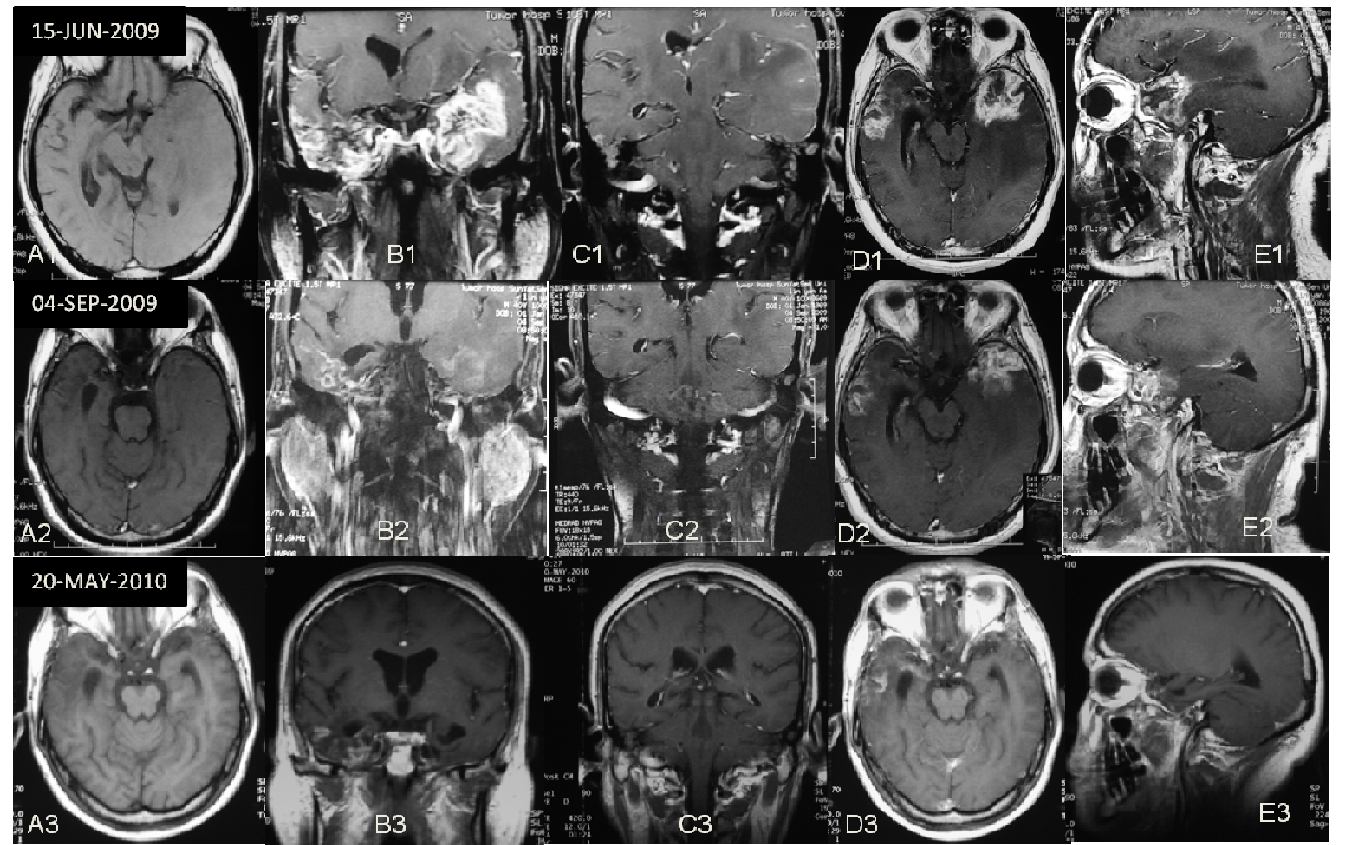

Fig. 2. Evolution of bilateral white matter lesions of a case in the anti-mycobacterium treatment (AMT) group before and after AMT. A1-E1: A1, left temporal lobe edema leads to compression of the brainstem before AMT; B1, postcontrast coronal T1-weighted MRI shows contrast-enhanced lesions in both temporal lobes; C1, coronal T1-weighted image shows a large edema lesion forming an irregular shape with finger-like projections in the left temporal lobe; D1, axial contrast-enhanced MRI shows enhancing lesions in both temporal lobes; E1, postcontrast sagittal T1-weighted MRI shows irregular mass of enhancement with perifocal edema. A2-E2: brain MRI shows regression of lesions on both sides after 3 months of AMT. A3-E3: lesions showed continuous regression in follow-up MRI examinations in May 2010.

Additionally, regions showing enhancement in the inferior treatment. Cysts, which suggest a later stage of TLN,6,10 temporal lobes in pretreatment MRIs disappeared with were also stabilized and decreased (Fig. 3).

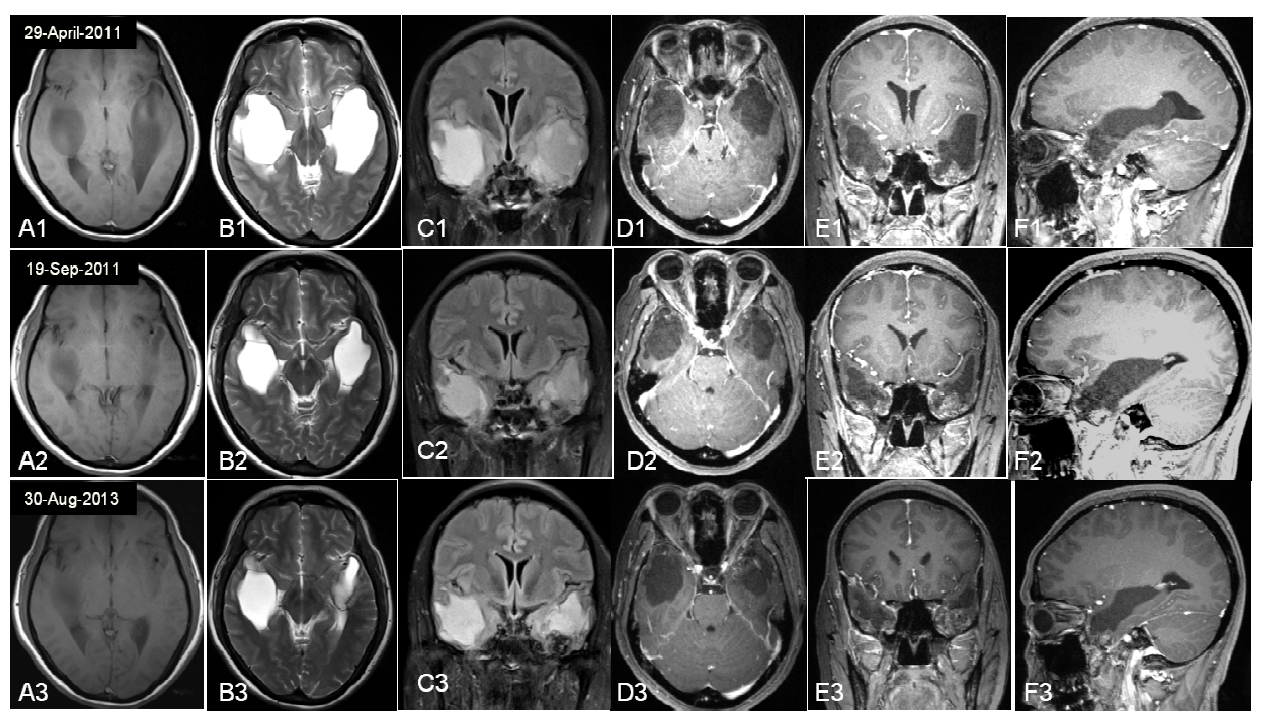

Fig. 3. Magnetic resonance evolution of temporal lobe cysts after anti-mycobacterium treatment (AMT) A1-F1: A1, axial T1-weighted; B1, axial T2-weighted; C1, coronal fluid-attenuated inversion-recovery (FLAIR) image shows bilateral temporal lobe cysts. Enhancing lesions in both temporal lobes were not obvious on postcontrast axial (D1), coronal (E1), or sagittal (F1) T1-weighted MRIs. A2-F2: MRIs show cysts in both temporal lobes that, 3 months after AMT, had decreased in size. A3-F3: An MRI performed 2 years later showed the right cyst regressed and the left static.

However, three patients showed expansion of lesions during Phase 2 of AMT, including one patient who was successfully treated (Fig. 4). 


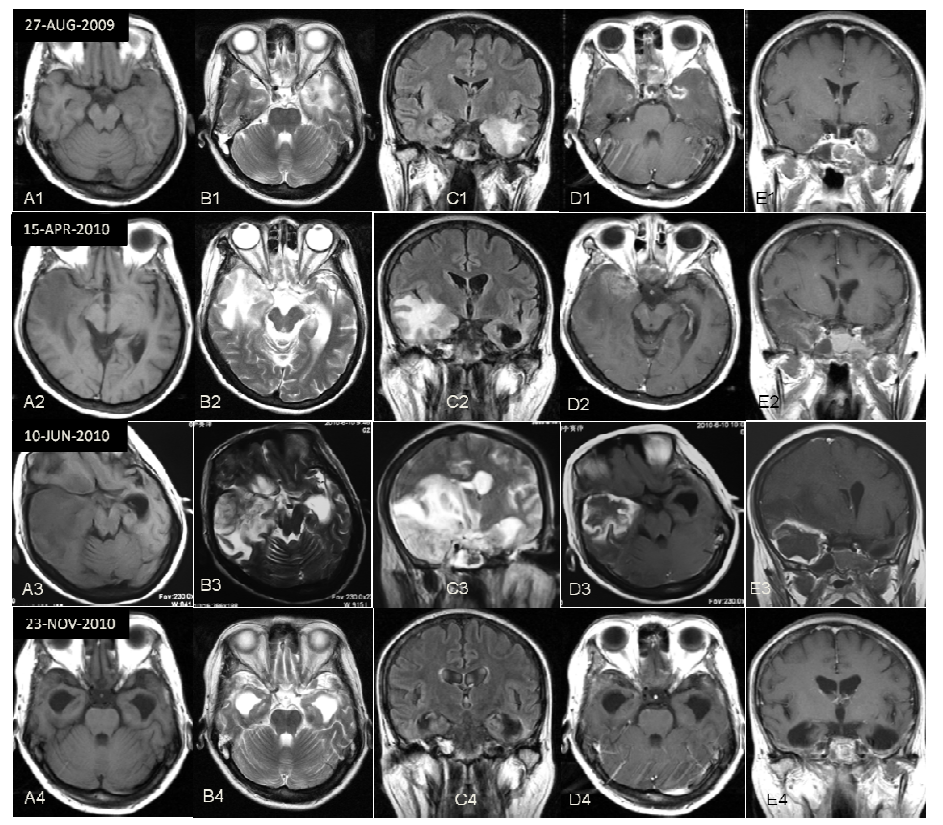

Fig. 4. Aberrant expansion of the lesions during anti-mycobacterium treatment (AMT). A1-E1: A1, left temporal lobe edema leads to mild compression of the brainstem on axial T1-weighted MRIs before AMT; B1, T2-weighted image shows an extensive lesion in the left temporal lobe and a small lesion in the right side; C1, coronal T2 fluid-attenuated inversion-recovery (FLAIR) image shows the left lesion; D1 and E1: enhanced lesions in both temporal lobes were found, which were more evident in the left lobe. A2-E2: subsequent MRI scan demonstrated an extensive white matter lesion in the inferior right temporal lobe with enhancement; the lesion showed a typical "Swiss cheese" pattern, whereas the lesion in the left temporal lobe had disappeared. A3-E3: MRI demonstrates enlargement of the lesion in the right temporal lobe with edema extending superiorly into the parietal lobe forming an irregular shape and finger-like projections and formation of a cavitating lesion with rim contrast enhancement. A4-E4: MRI shows complete regression of the lesions in the bilateral temporal lobes in November 2010.

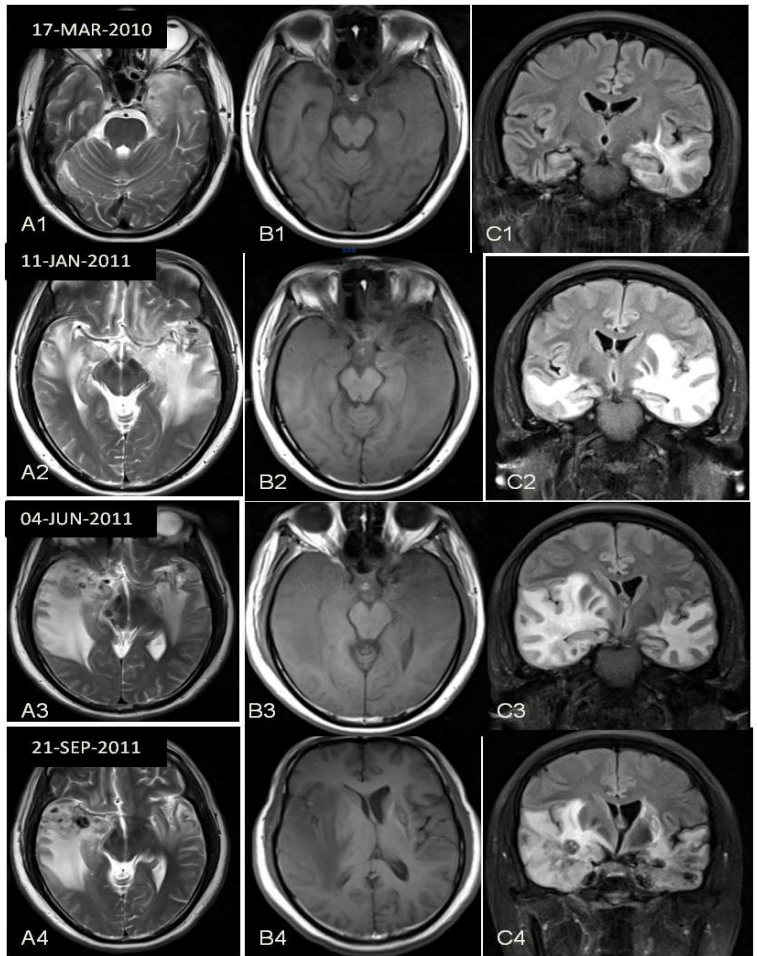

Fig. 5. Evolution of temporal lobe necrosis of a case in the control group. A1-C1: axial T2-weighted (A1), axial T1-weighted (B1), and coronal fluid-attenuated inversion-recovery (FLAIR) images show the appearance of mild white matter lesions (WMLs) bilaterally. A2-C2: MRIs show WMLs in both temporal lobes that, 10 months later, had increased in size. A3-C3: An MRI performed 6 months later showed a severe WML in the right temporal lobe with a large confluent area extending superiorly and causing a mass effect; however, the WML regressed on the left side. A4-C4: Given the patient's unexpected clinical decline, a follow-up brain MRI was performed in September 2011. The images revealed a modest increase in the size of the lesions with a "soap bubble" pattern.

In the control group, TLN lesions were generally persistent and became more severe and extensive over time (Fig. 5).

Only one patient showed mild reduction in the lesion size; however, that patient experienced chronically increasing difficulty in speaking and swallowing.

\subsection{Adverse Events in AMT Group}

Adverse events occurred in $7 / 10$ patients $(70 \%)$. Three patients had mild gastrointestinal syndrome on active therapy but were able to continue the trial and resolved quickly. Itching without rash was experienced by 2 patients. One patient experienced anorexia and stopped AMT. Two deaths that occurred in AMT group were considered related to the expansion of lesions.

\section{Discussion}

Here we report our initial experience with AMT for TLN, a severe type of DRI. This study shows that AMT can reverse and even clinically cure TLN. The patients enrolled in the study were in the advanced stage of TLN. The AMT effects were associated with significantly improved neurological status and changes in the temporal lobes observed by MRI. These results suggest that AMT is a potentially useful treatment for TLN. 
At present, there are no proven long-term treatment strategies for this significant radiation-induced late effect, and no prophylactic trial has been reported.11,12 Corticosteroids are used to reduce neurological symptoms and reduce brain edema in the short-term; however, long-term use is not generally advised because of the risk of immunosuppression and uncontrolled sepsis.13,14 Case reports in children and adults suggest a beneficial effect of hyperbaric oxygen, 15 but this finding remains to be conclusively demonstrated. Anticoagulants, 16 high-dose vitamins, and surgery have been attempted to combat DRI. However, none of these approaches has proven to be effective in controlled clinical trials. Bevacizumab, an antivascular endothelial growth factor antibody, has been used to treat symptomatic and progressive CNS radiation necrosis.17-19 However, the results of the latter studies were underpowered to detect significant changes in neurocognitive function and MRI changes due to small sample size with a short follow-up. To date, no patient has been reported to have complete resolution of both symptoms and MRI abnormality by these treatments. However, the findings of this study suggest that radiation necrosis of the brain can be successfully managed. All the patients receiving AMT responded nearly immediately to treatment. The worsening headaches, seizures, dizziness, and cognitive deterioration rapidly improved. AMT also ameliorated non-neurological complications, including xerostomia, foul odor, and trismus. Additionally, the effects of AMT were sustained. This study may provide a new approach for a pharmacological strategy by which to reverse progressive DRI.

The imaging findings of temporal changes following irradiation for nasopharyngeal carcinoma have been well documented.6,8 Cysts and bilateral WMLs represented late signs of TLN, which frequently became more severe and extensive over time and were associated with poor outcomes. There is no evidence in the literature that any treatment could effectively relieve these symptoms. However, ATM therapy resulted in striking regression of bilateral WMLs with contrast-enhanced necrotic lesions and cysts upon MRI, whereas patients receiving standard therapies showed progressive changes upon MRI. The remarkable changes observed in the imaging studies suggest that part of the treatment effect may be due to physiologic repair of radiation-damaged blood-brain barrier attained by decreasing the levels of inflammation through the use of AMT. To the best of our knowledge, AMT has provided the best results ever reported for DRI in humans.

The mechanisms by which the AMT interacts with TLN are not yet known. The idea that DRI may be radiation-induced inflammation should be addressed hypothetically. As early as 1934, Groff20 observed that injection of bacteria into the carotid artery or direct inoculation of bacteria into the brain of cats failed to induce brain abscess. Thus, the brain is normally resistant to infection. However, skull-base irradiation may unavoidably disrupt this function because of its CNS neurotoxicity, predisposing the included tissues to high risk of opportunistic mycobacterium infection. Several lines of evidence have shown that chronic inflammatory responses are associated with DRI.21-24 These results also provide a rationale for the use of anti-inflammatory-based interventions to prevent or ameliorate DRI. Although DRI may be a complex phenomenon that involves several molecular and cellular processes, our finding that AMT reverses TLN suggests that mycobacteria infection may be involved in the pathogenesis of DRI. The absence of a sensitive laboratory test for CNS mycobacterium infection might lead to failure to detect this type of infection. 25

Although AMT is promising, there are still many risks during the treatment. Three patients initially responded to AMT therapy, but their condition deteriorated with continued therapy. The mechanism responsible for this paradoxical phenomenon may be similar to that causing deterioration during treatment for CNS tuberculosis infection. It is well known that up to $10 \%$ patients with $\mathrm{CNS}$ TB after antituberculous treatment can show radiologic worsening of preexisting tuberculous lesions or develop a new lesion.[26-28] This paradoxical reaction can cause clinical deterioration or is just incidentally found when routine follow-up brain imaging is processed. It is believed that the paradoxical response is due to the enhancement of the immune response after active TB is controlled.[29] According to our limited experience, clinical improvement of the paradoxical expansion may be achieved through intravenous immunoglobulin and mannitol therapy. Besides that, Anti-tubercular drugs are similar to a lot of other medications in terms of causing side effects to the patients who took them. Examples of side effects caused by AMT consist of drug-induced hepatotoxicity, nephrotoxicity, peripheral neuropathy, hyponatremia,ototoxicity and rash. For this, careful patient monitoring clinically, hematologically and biochemically especially during first few weeks of AMT are necessary.

The study results must be interpreted within the context of possible limitations. We used an open-label, non-randomized study design in which a small number of patients was included; inevitably, this may cause potential sampling errors. Nevertheless, a nearly certain clinical diagnosis of TLN was attempted using careful patient selection, with the intent of excluding alternative differential diagnoses. Another study limitation is the lack of histologic documentation, due to the difficulties in obtaining histologic evidence of radiation-induced brain changes in patients who have undergone radiation therapy for nasopharyngeal carcinoma.

Despite these limitations, the striking facts that patients achieved complete resolution of both symptoms and MRI enhancement after the use of AMT lead us to believe that AMT could be a life-saving, cost-effective approach to combat cerebral radiation necrosis. These findings might also lead to prophylactic measures against radiation necrosis beyond what is already performed to control the effects of the radiation itself.

\section{Conclusions}

No firm conclusions can be drawn from this small 
prospective pilot study on a type of advanced DRI. However, our findings suggest that AMT may benefit patients suffering from the late effects of radiation on the nervous system. The results of this trial raise many questions, primarily about the precise mechanisms of action of the drugs used. Ongoing cellular and molecular studies and a randomized clinical trial will be needed to provide these answers.

\section{Financial Disclosure}

The authors declare that they have no competing financial interests. The sponsor of the study had no role in study design, data collection, data analysis, data interpretation, or writing of the report. The corresponding author had full access to all the data and had final responsibility for the decision to submit for publication.

\section{Study Funding}

This study was supported by grants from the Guangdong Natural Science Foundation (no. 2012B03180067)

\section{Author Contributions}

Yanqing Feng designed this study, analyzed the data, and take responsibility for the integrity of the data and the accuracy of the data analysis. Xi Chen, Ning Guo, Minying Zheng, Fan Huang, and Ke Ma collected data. Zhenhua Gao performed/supervised the majority of imaging studies. Yanqing Feng contributed to the writing of the manuscript. The study was supervised by Weixi Zhang.

\section{Acknowledgements}

The authors thank the patients for participating in this study. This study was supported by a grant from the Guangdong Natural Science Foundation (grant no. 2012B03180067).

\section{References}

[1] Sheline GE, Wara WM, Smith VS. Therapeutic irradiation and brain injury. Int J Radiat Oncol Biol Phys 1980; 6(9): 1215-28.

[2] Fischer AW, Holfelder H. Lokales amyloid in gehirn. Dtsch Z Chirurg. 1930; 227: 475-83.

[3] Soussain C, Ricard D, Fike JR, Mazeron JJ, Psimaras D, Delattre JY. CNS complications of radiotherapy and chemotherapy. Lancet 2009; 374(9701): 1639-51.

[4] Lee AW, Law SCK, $\mathrm{Ng} \mathrm{SH}$, et al. Retrospective analysis of nasopharyngeal carcinoma treated during 1976-1985: late complications following megavoltage irradiation. $\mathrm{Br} J$ Radiol 1992; 65(778): 918-28.

[5] Valk PE, Dillon WP. Radiation injury of the brain. Am J Neuroradiol 1991; 12(1): 45-62.

[6] Lee $\mathrm{AW}, \mathrm{Ng} \mathrm{SH}$, Ho JH, et al. Clinical diagnosis of late temporal lobe necrosis following radiation therapy for nasopharyngeal carcinoma. Cancer 1988; 61(8): 1535-42.

[7] King AD, Ahuja AT, Yeung DK, et al. Delayed complications of radiotherapy treatment for nasopharyngeal carcinoma: imaging findings. Clin Radiol 2007; 62(3): 195-203.

[8] Chong VE, Fan YF. Radiation-induced temporal lobe necrosis. Am J Neuroradiol 1997; 18(4): 784-5.

[9] Cheng KM, Chan CM, Fu YT, et al. Brain abscess formation in radiation necrosis of the temporal lobe following radiation therapy for nasopharyngeal carcinoma. Acta Neurochir (Wien) 2000; 142(4): 435-40.

[10] Wang YX, King AD, Zhou $H$, et al. Evolution of radiation-induced brain injury: MR imaging-based study. Radiology 2010; 254(1): 210-8.

[11] Greene-Schloesser D, Robbins ME, Peiffer AM, et al. Radiation-induced brain injury: a review. Front Oncol 2012; 2: 73.

[12] Kim JH, Brown SL, Jenrow KA, et al. Mechanisms of radiation-induced brain toxicity and implications for future clinical trials. J Neurooncol 2008; 87(3): 279-86.

[13] Tada E, Matsumoto K, Kinoshita K, et al. The protective effect of dexamethasone against radiation damage induced by interstitial irradiation in normal monkey brain. Neurosurgery 1997; 41(1): 209-17.

[14] Martins AN, Severance RE, Henry JM, et al. Experimental delayed radiation necrosis of the brain. Part 1 . Effect of early dexamethasone treatment. J Neurosurg 1979; 51(5): 587-96.

[15] Chuba PJ, Aronin P, Bhambhani K, et al. Hyperbaric oxygen therapy for radiation-induced brain injury in children. Cancer 1997; 80(10): 2005-12.

[16] Glantz MJ, Burger PC, Friedman AH, et al. Treatment of radiation-induced nervous system injury with heparin and warfarin. Neurology 1994; 44(11): 2020-7.

[17] Gonzalez J, Kumar AJ, Conrad CA, et al. Effect of bevacizumab on radiation necrosis of the brain. Int $J$ Radiat Oncol Biol Phys 2007; 67(2): 323-6.

[18] Levin VA, Bidaut L, Hou P, et al. Randomized double-blind placebo-controlled trial of bevacizumab therapy for radiation necrosis of the central nervous system. Int J Radiat Oncol Biol Phys 2011; 79(5): 1487-95.

[19] Jeyaretna DS, Curry WT Jr, Batchelor TT, et al. Exacerbation of cerebral radiation necrosis by bevacizumab. J Clin Oncol. 2011; 29(7): e159-62.

[20] Groff RA. Experimental production of abscess of the brain in cats. Arch Neurol Neurosurg Psychiatry 1934; 31: 199-204.

[21] Ballesteros-Zebadúa P, Chavarria A, Celis MA, et al. Radiation-induced neuroinflammation and radiation somnolence syndrome. CNS Neurol Disord Drug Targets 2012; 11(7): 937-49.

[22] Monje ML, Mizumatsu S, Fike JR, et al. Irradiation induces neural precursor-cell dysfunction. Nat Med 2002; 8(9): 955-62.

[23] Rola R, Sarkissian V, Obenaus A, et al. High-LET radiation induces inflammation and persistent changes in markers of hippocampal neurogenesis. Radiat Res 2005; 164(4 Pt 2): 556-60. 
[24] Ramanan S, Kooshki M, Zhao W, et al. The PPARalpha agonist fenofibrate preserves hippocampal neurogenesis and inhibits microglial activation after whole-brain irradiation. Int $J$ Radiat Oncol Biol Phys 2009; 75(3): 870-7.

[25] Feng Y, Guo N, Liu J, et al. Mycobacteria infection in incomplete transverse myelitis is refractory to steroids: a pilot study. Clin Dev Immunol 2011; 2011: 501369.

[26] Cheng VC, Ho PL, Lee RA, et al. Clinical spectrum of paradoxical deterioration during antituberculosis therapy in non-HIV infected patients. Eur J Clin Microbiol Infect Dis 2002; 21(11): 803-9.
[27] Afghani B, Lieberman JM. Paradoxical enlargement or development of intracranial tuberculomas during therapy: case report and review. Clin Infect Dis. 1994;19(6):1092-9.

[28] Thwaites GE, Macmullen-Price J, Tran TH, et al. Serial MRI to determine the effect of dexamethasone on the cerebral pathology of tuberculous meningitis: an observational study. Lancet Neurol. 2007;6(3):230-6.

[29] Nicolls DJ, King M, Holland D, et al. Intracranial tuberculomas developing while on therapy for pulmonary tuberculosis. Lancet Infect Dis. 2005;5(12):795-801. 